\title{
Políticas de promoción y estímulo de la innovación: La importancia de la implementación de instrumentos
}

Promoting scientific and technological innovation through public policies: the relevance of instruments

Victoria Castro Demiryi

Mg. en Política y Gestión de la Ciencia

y la Tecnología, Facultad de Ciencias

Económicas, UBA.

IHUCSO Litoral

(CONICET-UNL) - Instituto de

Humanidades y Ciencias Sociales del Litoral vico1517@gmail.com
Fecha de recepción:

29.7.17

Fecha de aceptación:

8.10.18

\section{Resumen}

El trabajo analiza las características del proceso de implementación de instrumentos de política pública de promoción en ciencia, tecnología e innovación (CTI), desde los modelos de gestión y el enfoque Ciencia, Tecnología y Sociedad. La indagación se realiza a la luz de un instrumento particular implementado en 2006 por la Agencia Nacional de Promoción Científica y Tecnológica Argentina. El denominado Programa de Áreas Estratégicas (PAE) fue el primer instrumento asociativo puesto en marcha en el marco de la política de integración y fortalecimiento del Sistema Nacional CTI. El análisis de la dinámica implementación y su revisión a la luz de los objetivos de política científica (science policy) puede significar un aporte al estudio de la gestión pública en el sector; a partir de la posibilidad de utilizar la información recogida como insumo para la evaluación de resultados y el diseño de herramientas de gestión acordes a los instrumentos diseñados. 
Palabras clave: políticas públicas - sistema nacional de innovación, - CTI - política de ciencia y técnica - República Argentina.

Abstract: The paper focuses on the implementation process of public policy instruments for the promotion of science, technology and innovation, through the implementation of management models and the Science, Technology and Society approach. The inquiry is conducted under the light of a particular instrument implemented in 2006 by Argentina's National Agency for Scientific and Technological Promotion. The so-called Strategic Areas Program (PAE) was the first associative instrument put in place within the framework of the policy of integration and strengthening of the National System of science, technology and innovation. The analysis of its dynamic implementation through the light of the scientific policy objectives (science policy) is pretended to be a contribution to the study of policy management in the sector; from the input of the information as input for the evaluation of the results and the design of management tools.

Keywords: Public policies - national system of innovation - CTI - science and technological policies - Argentina

\section{Introducción}

El siglo XXI ha dado paso a perspectivas y discusiones que surgen como respuesta a consecuencias derivadas de ciertos usos, aplicaciones y consecuencias de las ciencias. El quehacer de la ciencia se ha vuelto más complejo e intrincado, incorporando la tecnología y la innovación en un proceso amplio, interactivo, dialogado, participativo y reflexivo, no centrado en la calidad científica como fuente exclusiva de legitimidad. Los modos de hacer ciencia se han profundizado, modificado y estimulado, entre otras cosas, por la necesidad de solucionar problemas que exceden la consideración propia y exclusiva de los investigadores.

El cambio en el esquema de producción de conocimiento presenta un correlato en las políticas denominadas de Ciencia,Tecnología e Innovación (Albornoz, 2001) introduciendo la necesidad de que las mismas se orienten a estimular la demanda de conocimientos y aplicación eficiente de 
nuevos conocimientos, e innovación (Zurbriggen y González Lago, 2010:23) a través del diseño de acciones de promoción que incorporan e involucran nuevos y diferentes actores al proceso.

Asimismo, los cambios en el diseño de instrumentos y/o herramientas, destinados a la promoción de la ciencia y la tecnología, se evidencian al momento de su aplicación o puesta en marcha, en la etapa denominada implementación ${ }^{1}$.

Desde la ciencia política la preocupación por cómo los gobiernos toman decisiones, cómo las ponen en práctica, por qué se buscan ciertas alternativas y no otras, se podría decir que son problemáticas recientes en el campo. Hace apenas unas décadas tomó relevancia a nivel científico la comprensión del accionar gubernamental, no tanto desde la idea de estado como máxima institución, ni los sistemas políticos o los regímenes; son los gobiernos los que cobran sentido como objeto de estudio, dando lugar a una rama de la ciencia política: las políticas públicas o estatales. Su estudio permite "una visión del estado en acción", desagregado y descongelado como estructura global y “puesto en un proceso social”, tal como sostienen Oszlak y O`Donnell (1994:104).

Las acciones del estado son parte de un proceso histórico e interactivo que involucra tanto la toma de decisión respecto de una cuestión determinada, su tratamiento, su resolución, y los procesos burocráticos que se inician al interior del mismo. Estos procesos desencadenan consecuencias y forman parte de la ejecución ("implementación”) de la política. Así una política estatal se constituye en "nudos" en una secuencia de interacciones de la que participan diferentes actores sociales, entre ellos los actores de la burocracia estatal y los actores beneficiarios de esa política.

\section{La importancia de los estudios de la implementación de políticas}

En el marco de los estudios de las políticas públicas, diferentes autores llaman la atención durante la década de 1970 respecto de la implementación como campo de investigación ${ }^{2}$. La importancia de los estudios de implementación adquiere relevancia cuando los especialistas comienzan a poner el foco en la existencia de una brecha entre los objetivos propuestos y los resultados alcanzados (Revuelta Vaquero 2007). Hasta entonces la reflexión se centraba en la formulación y la política pública era definida como la toma de decisión o la adopción de una alternativa, en tanto que la implementación aparecía como una cuestión técnica que no formaba parte de la política (Bañon y Carrillo, 1997).

Zurbriggen y González Lago sostienen, con relación a los estudios de las políticas públicas de ciencia, tecnología e innovación en América Latina, que éstas tuvieron un punto de inflexión a fines de la década de 1990, a partir de la reestructuración de los aparatos del estado y la incorporación de marcos conceptuales ${ }^{3}$ con foco en las instituciones. En 1990 fue sancionada en Argentina la Ley de Promoción y Fomento de la Innovación Tecnológica (Ley 23.877) que estableció el marco normativo para la implementación de instrumentos destinados al impulso, la promoción y el estímulo de la innovación. Así, las políticas del sector abren paso a una nueva institucionalidad, centrada en el apoyo a la innovación, el desarrollo tecnológico y el mejoramiento de las capacidades estatales. 
En el marco de una política económica diferente a la que rigió en el país en los orígenes de la nueva institucionalidad, a saber: promoción industrial, tipos de cambio múltiples, re-estatización de empresas, apertura comercial administrada y regulación de mercados, desde el año 2005 los recursos de apoyo a la innovación se expanden, al mismo tiempo que se diversifican los instrumentos promocionales, específicamente desde 2007.

(Del Bello y Abeledo 2007:1-17).

La reforma institucional en la política de promoción de la innovación se vio expresada en nuestro país en la creación de organismos y el aumento de recursos económicos -sobre todo provenientes de entes internacionales-,${ }^{4}$ en la elaboración de planes estratégicos; en el fortalecimiento de las capacidades institucionales de los encargados de elaborar e implementar políticas y en el diseño de nuevos instrumentos.

En 2011 un documento de la Organización para la Cooperación y el Desarrollo Económicos (OCDE) destacó el decidido avance de los gobiernos latinoamericanos en el establecimiento de políticas e instituciones que potencian las capacidades de ciencia, tecnología e innovación. ${ }^{5}$ Como parte de esos esfuerzos, señaló la necesidad de aprender a diseñar e implementar políticas de innovación capaces de acompañar el proceso de cambio, adaptándose a un contexto que requiere cada vez más eficacia, eficiencia y efectividad de las acciones de política pública. Entre las debilidades estructurales que el documento de la OCDE señaló se encuentra la "escasa capacidad de monitoreo y evaluación de los programas implementados, insuficiente retroalimentación entre el diseño y la implementación” (OCDE 2011:19).

En Argentina, la dimensión de la implementación, junto con la del diseño y la formulación comienza a incorporarse al discurso de las políticas públicas a principios de la década actual. Los ámbitos de planificación de políticas de ciencia, tecnología e innovación de Argentina ponen atención a esta cuestión de la implementación del Ministerio de Ciencia, Tecnología e Innovación (MINCYT) afirmaba en 2012 que

las políticas públicas en un Estado democrático, pluralista $y$ profundamente inclusivo, encuentran su mayor fortaleza y legitimación cuando en su formulación e implementación participan activamente los diferentes actores de la sociedad que serán afectados por ellas

(Ladenheim 2012).

La pretensión de estudios desde un enfoque que analice los cursos de acción posteriores a la formulación como un aspecto más del proceso de las políticas públicas requiere del 
estudio de casos como estrategia de investigación.Asimismo, los estudios de implementación pueden significar un aporte a la gestión de la ciencia y la tecnología, a partir del análisis ex post de la puesta en marcha de instrumentos o herramientas de política estatal para el sector.

\section{Los estudios sociales de la ciencia como marco de análisis}

El campo de los estudios sobre ciencia, tecnología y sociedad, denominado CTS $^{6}$ se vincula con la necesidad de profundizar sobre las relaciones entre los grupos humanos, y los conocimientos y prácticas que generan y desarrollan respecto de los ámbitos que el título comprende. En el campo de la política, los estudios CTS, en términos generales, han defendido una activa participación pública en la gestión de la ciencia y la tecnología, "promoviendo la creación de diversos mecanismos institucionales, que faciliten la apertura de los procesos de toma de decisiones en cuestiones concernientes a políticas científico-tecnológicas”. (López Cerezo, González García y Luján 1996:12). Desde las distintas disciplinas y los enfoques que lo integran se intenta analizar de manera crítica el complejo Ciencia y Tecnología, cuestionando fundamentalmente las visiones que lo desarraigan de los contextos humanos -sociales, políticos, económicos, culturales- en los cuales surge, se desarrolla y respecto de los que se transforma e impacta.

Los estudios CTS nacen de la convergencia de diversas trayectorias profesionales y académicas preocupadas por el análisis de la ciencia como actividad social y en su contexto (Edge, 1995). ${ }^{7}$ Historiadores, filósofos, sociólogos, antropólogos, economistas y politólogos confluyen con sus respectivas tradiciones en una postura crítica hacia la ciencia y la tecnología, claramente contrapuesta de aquella visión optimista del progreso científico heredada de la Segunda Guerra Mundial (López Cerezo, 1998:41-68) y representada en la institucionalización del modelo lineal.

En América Latina, a finales de los años sesenta se gestó un ámbito de pensamiento concentrado en el cuestionamiento a ciertos supuestos básicos respecto de la producción de la ciencia y tecnología; más tarde estas críticas se reunieron bajo la denominación de Pensamiento Latinoamericano en Ciencia,Tecnología y Sociedad (PLACTS). ${ }^{8}$ Puntualmente, la reflexión se focalizaba en la estructura y el carácter social de la ciencia y la tecnología y de las políticas implementadas. ${ }^{9}$ Una de las críticas más fuertes del pensamiento latinoamericano está dirigida al denominado modelo lineal, su concepción respecto de la generación de conocimiento y su aplicabilidad, como el producto de una sucesión de etapas bien definidas donde en cada una de ellas se realiza una parte del trabajo sin demasiada conexión con el resto. Este tipo de configuración respondía a una concepción, que podría calificarse de ofertista y que, en América Latina, fue impulsada por la Organización de las Naciones Unidas para la Educación, la Ciencia y la Cultura (UNESCO) en la década de 1950, donde se pretendía fortalecer las instituciones de investigación, con la idea de que éstas generarían derrames al sector productivo, bajo el presupuesto implícito de que la oferta crea su demanda. 
Una de las líneas de análisis promovida por la organización mencionada sostenía que la mayoría de los países latinoamericanos cargan con la herencia del modo de producción científico-tecnológico de los países europeos. Este modelo generó controversias sobre la utilidad y aplicación de los resultados de la investigación y desarrollo para la resolución de problemas productivos y sociales, poniendo de manifiesto la dificultad para constituir una infraestructura científico-tecnológica -en términos de Sábato- que funcione como un verdadero Sistema Nacional de Ciencia y Tecnología.

Así, el denominado PLACTS nace no sólo como una crítica a la situación de la ciencia y la tecnología, sino también de algunos aspectos de la política estatal impulsados en parte por sugerencias como las de UNESCO.

Por otra parte, puede identificarse una segunda línea de reflexión en el marco de los estudios CTS relacionada con los estudios de innovación desde la perspectiva de la economía evolucionista. Esta línea está dirigida al estudio del rol de las tecnologías en las empresas, más que a la orientación de políticas. Desde la visión evolucionista del cambio técnico, se pretende describir los procesos de aprendizaje, adaptación y trayectorias tecnológicas. Esta perspectiva introduce al campo de estudio el concepto de Sistemas Nacionales de Innovación (SNI) puesto en circulación por Bengt-Ake Lundvall en 1992, entendiendo este como una red compleja de actores, instituciones y funciones relacionadas en procesos producción, difusión y uso de nuevos conocimientos, con capacidades complementarias e intereses diferenciados pero susceptibles de articulación.

Los estudios de innovación aportaron durante los años noventa a la construcción del discurso de la política científica y tecnológica. Uno de los resultados de esos aportes ha sido el pasaje conceptual de Sistema Nacional de Ciencia y Tecnología a Sistema Nacional de Innovación, incorporando de manera plena el concepto de innovación (Vacarezza 1998:31) y justificando la creación de instrumentos que garantizaran su funcionamiento.

Indudablemente, el campo $\mathrm{CTS},{ }^{10}$ a pesar de sus limitaciones y transferencias geográficas, se presenta como posibilidad de abordaje, tanto como espacio de intervención en el ámbito de la política de ciencia, tecnología e innovación. Asimismo, podría ser entendido y presentado como marco de acción para el ámbito de la gestión donde esas políticas se implementan. Esto solo es posible en la medida en que entendamos la gestión como parte de la política y no como actividad escindida y tarea exclusiva de cuadros técnicos administrativos.

\section{Modelos de Innovación: La noción de Sistemas Nacionales de Innovación}

La noción de Sistemas Nacionales de Innovación (SNI) surge frente a la necesidad de explicar las diferencias de crecimiento entre países y entre sectores productivos, como 
enfoque alternativo a las teorías tradicionales de la economía de la innovación y ante sus limitaciones para dar cuenta de esas diferencias.

La definición conceptual de SNI varía según los autores. Para Freeman (1987) se trata de “...la red de instituciones en los sectores público y privado cuyas actividades e interacciones inician, importan, modifican y difunden nuevas tecnologías". Lundvall (1992), entiende el SNI como “...los elementos y relaciones que interactúan en la producción, difusión y uso de conocimientos nuevos y económicamente útiles... y se localizan dentro o en las fronteras de un Estado”. Nelson (1993) lo define como “... una serie de instituciones cuya interacción determina la capacidad innovadora... de las empresas de un país". Patel y Pavitt (1994), por su parte, dan cuenta del mismo a partir de aquellas "instituciones nacionales, sus estructuras de incentivos y sus competencias que determinan la velocidad y la dirección del aprendizaje tecnológico (o el volumen y composición de las actividades generadoras de cambio) en un país".

A modo de síntesis podemos rescatar que todos los autores comparten en sus definiciones la idea de interacción, de intercambio, algunos resaltando el papel del Estado y otros en el papel de las empresas. Al margen de las variaciones en su definición, podemos concebir el SNI como un espacio de construcción, de aprendizaje social. Un proceso -del cual el desarrollo tecnológico es parte- para la generación e intercambio de flujos de información y conocimientos entre los diversos agentes nacionales y regionales, en búsqueda de incrementar la productividad y la competitividad de los sectores productivos, la generación de empleo y el mejoramiento de la calidad de vida de la población. Los diferentes enfoques de los sistemas de innovación comparten el énfasis en la nolinealidad y la heterogeneidad en la producción de conocimiento. Asimismo, todas las conceptualizaciones en torno a la noción de SNI ponen el acento en el papel que cumplen las instituciones científicas y educativas en el proceso de desarrollo productivo, a través de la innovación tecnológica.

Johnson y Lundvall (1994) señalan dos aspectos importantes en torno a la noción de SIN: a) la dimensión nacional/regional de los sistemas de innovación, señalando que la mayor importancia que se da al sistema nacional no excluye el análisis de los sistemas de innovación locales, regionales o transnacionales; b) el carácter sistémico como una idea que supera lo institucional o una actividad en particular; el término "sistema" se refiere a una manera de articular diversas instituciones y actividades en ese proceso de aprendizaje que tiene como objetivo la identificación de posibilidades de innovación y su consecuente realización.

El concepto de Sistema Nacional de Innovación adquiere relevancia desde mediados de la década de 1980, a partir de la expansión en el uso y aplicación de la noción en sí misma como SNI. Lundvall (2002) sugiere que la legitimación que ha ganado dicha teoría se debe, en parte, a que la teoría y la política difundida por el "mainstream” ha 
sido insuficiente a la hora de explicar los factores que influyen en la competitividad y su relación con el desarrollo económico.

En los últimos años, se ha prestado atención al concepto en niveles diferentes al nacional, aludiendo a la noción de "Sistemas Regionales". Se ha observado que la estructura de los sistemas nacionales de producción e innovación es el resultado de un proceso histórico y no puede ser transferido tan fácilmente. Las relaciones entre los diferentes elementos son importantes para el funcionamiento del sistema como un todo.Adquiere relevancia entonces la estructura de los Sistemas Regionales de producción e innovación como etapa importante en el estudio del SNI. ${ }^{11}$

Es de destacar, asimismo, que el enfoque del SNI supone un rol particular por parte del Estado -en el marco de las políticas públicas- en relación con la idea de aprendizaje por interacción. La institucionalización del proceso de aprendizaje se encuentra condicionada por cada estructura económica e institucional particular y puede producirse a través de la elaboración de políticas.

\section{Modelos e institucionalización en Argentina}

Retomando la línea del PLACTS, ${ }^{12}$ autores como Carrizo y Alfonso (2013:96-105), plantean que el crecimiento y consolidación de la ciencia y la tecnología en países periféricos fueron acompañados de la aplicación de modelos teóricos importados que guiaron la formulación de políticas. Así, en la bibliografía especializada se pone en discusión si las dificultades para el desarrollo de la ciencia y la tecnología en Argentina se deben al modelo lineal y las "políticas de oferta", ${ }^{13}$ a la desarticulación entre las instituciones dedicadas a la ciencia y la tecnología, o a la falta de inversión por parte del sector privado, entre otros argumentos.

La concepción de tipo ofertista impulsada por UNESCO es contemporánea con la creación en nuestro país de grandes instituciones destinadas a la producción de ciencia y tecnología. Durante la década de 1950 se crearon la Comisión Nacional de Energía Atómica (CNEA), el Instituto Nacional de Tecnología Industrial (INTI), el Instituto Nacional de Tecnología Agropecuaria (INTA), y el Consejo Nacional de Investigaciones Científicas y Técnicas (CONICET). Bisang (1997) plantea que si bien estas instituciones contribuyeron a modificar el panorama tecnológico local, falló la implantación de un modo de funcionamiento sistémico, producto de un denominador común: el haber sido la respuesta a problemas puntuales o sectoriales pero carentes de articulación interinstitucional, exponiendo como resultado de este proceso altamente complejo un importante grado de desarrollo del sistema científico y tecnológico pero con pocos vínculos entre sí. 
Otro de los organismos internacionales que ha cumplido un papel importante en cuanto a los enfoques de política científica que los gobiernos de América Latina han seguido, es la OCDE, que en la década de 1960 publica un documento con recomendaciones para los gobiernos respecto del apoyo a la investigación científica y tecnológica. ${ }^{14}$ En el análisis de Elzinga y Jamison (citados en Albornoz, 2007), lo más importante del documento fue que transformó una ambición política o un enfoque en una doctrina de política estratégica: la idea de que la ciencia, junto con la educación superior, debían ser consideradas como un factor productivo en pie de igualdad con el trabajo y el capital, en la búsqueda del crecimiento económico. En los años ochenta, la OCDE presentó un nuevo documento (OCDE 1981) ${ }^{15}$ donde se estimulaba el desarrollo de nuevas tecnologías y se intentaba acercar las empresas a las universidades y viceversa. También se empezó a prestar atención a la teoría de la innovación, como parte de la búsqueda de nuevos marcos conceptuales que permitieran orientar la reestructuración económica y el fortalecimiento de la competitividad. A partir de esta década se expande y fortalece, tanto en Europa como en los Estados Unidos, la generación de estudios dentro del marco conceptual del SNI.

Algunos autores avocados al estudio de los problemas del sector científico y tecnológico y a las implicancias para la política del sector, sostienen que la mayoría de los países latinoamericanos cargan con la herencia del modo de producción científico-tecnológico de los países centrales. ${ }^{16}$ Este modelo generó controversias sobre la aplicabilidad y utilidad de los resultados de la Investigación y Desarrollo (I+D) para la resolución de problemas productivos y sociales, poniendo de manifiesto la dificultad para constituir SNI que funcionen como tales. En este sentido, Argentina no fue la excepción. Desde el nacimiento mismo de las instituciones de ciencia y tecnología en nuestro país existieron intensas discusiones sobre las relaciones entre los sectores que intervenían en la producción científico-tecnológica y en el proceso de innovación. Uno de los resultados de este debate fue la noción de "triangulo de Sábato", que no hace más que aludir a la característica sistémica de un proceso de innovación.

El autor argentino Jorge Sábato elaboró un sencillo modelo con tres elementos básicos: el gobierno $(\mathrm{G})$, la estructura productiva (E) y la infraestructura científico-tecnológica (I). Entre estos componentes, se establece una serie de interrelaciones, dando como resultado una estructura de relaciones con forma similar a la de un triángulo. En el vértice Infraestructura científica-tecnológica, se encuentra la capacidad creadora, ya que el autor considera que la investigación es producto de la inteligencia humana; el vértice Estructura productiva está compuesto por todos los sectores productores de bienes y servicios y, por último, el vértice Gobierno comprende las redes y roles institucionales, cuya función es canalizar los recursos, implementar políticas e intermediar entre los dos vértices anteriores.

En este esquema, denominado “Triángulo IGE”, el autor plantea la necesidad de vincular a los sectores científico y tecnológico, estatal y productivo, para lograr el desarrollo. 
Esta idea pone en tensión la noción de producción de conocimiento e innovación como instancias separadas o estancas, e introduce la posibilidad de pensarla como proceso desordenado, interactivo y que implica feed back. Se configura así el esquema de interacciones entre estos tres sectores, que no es otra cosa que el "sistema" en el que están insertas las actividades de innovación, generando un proceso de retroalimentación entre agentes e instituciones y, obteniendo como resultado una estrategia de desarrollo propia, acorde a las características propias del territorio del que se trate.

Entendido de esta manera, puede decirse que cada país configura diferentes sistemas en los que estas relaciones se ordenan de alguna manera. La tarea, entonces, no sería montar un sistema de innovación, sino mejorar, fortalecer y dinamizar los vínculos de los sistemas existentes, según las características que estos adquieran en cada caso.

\section{Las Políticas Públicas como proceso}

En el enfoque relacional de las políticas públicas (H. Laswell, 1960), éstas se presentan como el resultado de una compleja y conflictiva vinculación entre actores del Estado y la sociedad. Es más completo y dinámico, involucra a los diferentes actores, da cuenta de su diversidad, acuerdos y rupturas tanto en la sociedad como en el Estado, pero a la vez -sostiene Miró ${ }^{17}$ - reconoce la acción estatal como instancia donde se deciden e instrumentan las políticas públicas.

El estudio y análisis de las políticas públicas ofrece enfoques y definiciones en permanente transformación y adecuadas a diferentes contextos. ${ }^{18}$ Según Tamayo Sáez las políticas públicas son "el conjunto de objetivos, decisiones y acciones que lleva a cabo un gobierno para solucionar los problemas que en un momento dado el gobierno y los ciudadanos consideran prioritarios" (Tamayo Sáez 1997:281). Desde este punto de vista, las políticas públicas pueden ser entendidas como un proceso, sostiene el autor, en el que confluyen "acciones y decisiones" que toman los gobiernos para satisfacer necesidades y demandas, y en las que adquiere relevancia la participación de la ciudadanía en su consecución.

El estudio de las políticas públicas ha derivado a partir de los años cincuenta en modelos que dan cuenta de las mismas como un proceso, ciclo o sistema. ${ }^{19} \mathrm{El}$ enfoque actual, sostiene Pallares (1986:150) tiene sus orígenes en los estudios sobre el proceso decisional y los realizados durante los años '60, puntualmente sobre la elaboración de políticas (policy-making). En este sentido, el autor plantea que actualmente los estudios dedicados a las políticas públicas concentran los aportes del modelo "racional" de Simon (1947, 1960 y 1983), y el "incremental”, de Lindblom $\left(1959,1968\right.$ y 1979), que anteriormente polarizaban el debate. ${ }^{20}$ 
El modelo para el abordaje de las políticas públicas tuvo en los años setenta el aporte de autores como Jones y Anderson, quienes sobre la idea de sistema, identifican etapas y actividades para un proceso -el de las políticas públicas- que tiene una dinámica propia. ${ }^{21}$

Retomando la definición de Tamayo Sáez las políticas públicas pueden entenderse como un proceso que se inicia cuando un gobierno o un directivo público detecta la existencia de un problema que, por su importancia, merece su atención y termina con la evaluación de los resultados que han tenido las acciones emprendidas para eliminar, mitigar o variar ese problema (Tamayo Sáez 1997:281).

De este modo, el ciclo comienza cuando un asunto es problematizado y a partir del cual el Estado decide intervenir a través de una determinada política pública. ${ }^{22}$ Así, la cuestión va atravesando diferentes momentos, fases o etapas desde su problematización hasta su resolución.

Las etapas cualquier política pública, recorre son, según Tamayo Sáez: la identificación, definición del problema y estructuración de la agenda; la formulación de las alternativas; la adopción de una alternativa, la implementación, y la evaluación.

1) Definición del problema, detección y estructuración de la agenda: esta etapa consiste en "identificar a los actores con algún tipo de intereses en el problema y reconstruir sus definiciones, la forma en que ven el problema. Desde este punto de vista, la definición de problemas es ante todo una cuestión política". (Tamayo Sáez 1997:284). Esta fase se completa con la detección del problema por parte de los actores responsables desde la administración y con el diseño de la agenda. En relación al primer punto, son los decisores públicos quienes deben garantizar la interacción con el entorno, la participación de actores públicos y privados en el proceso, y la recolección de información para la detección del problema; tanto a través de instrumentos diseñados para tal fin dentro de la misma organización administrativa; como también la información generada en instancias de evaluación de experiencias anteriores. Por otra parte, no todos los problemas que se visibilizan en la sociedad poseen la misma prioridad para la administración pública. Tamayo Sáez (1997) llama Agenda Sistémica a los problemas que preocupan a la sociedad en un momento determinado, y Agenda Institucional o Política como la evolución de la primera y la inclusión de los problemas en ésta es condición para convertirse en política pública. El ingreso a la Agenda Política se ve influenciado por diferentes tipos de factores como el poder e influencia de los grupos de interés, barreras culturales y a la innovación. Asimismo, existen factores como los medios de comunicación, las necesidades de crecimiento institucional y el comportamiento de los decisores, entre otros, tendientes a contrarrestar los primeros. La Agenda Política generalmente comporta problemas viejos y algunos nuevos, esa acumulación de problemas viejos y 
recurrentes se debe a que los problemas públicos en muy pocas ocasiones tienen una solución definitiva; más bien al contrario, el impacto de las políticas consigue aminorar o eliminar unas dimensiones del problema, poniendo en evidencia la existencia de otras dimensiones. (Tamayo Sáez 1997:286);

2) Formulación de las alternativas: ${ }^{23}$ esta etapa incluye el establecimiento y priorización de objetivos, la generación de alternativas para alcanzarlos, la valoración de las consecuencias de las distintas opciones generadas y la adopción de una o varias de esas alternativas posibles;

3) Adopción de una alternativa: esta fase se encadena con la anterior y supone la opción o combinación de alternativas maximicen los objetivos determinados.

4) Implantación: la etapa de implantación o implementación supone la materialización y aplicación de la alternativa seleccionada.

5) Evaluación: esta fase se constituye en el último estadio y el reinicio del ciclo ya que del análisis de los resultados surge una nueva propuesta, su continuación o su desestimación. Esta propuesta no resulta ser solo una herramienta técnica, sino también política ya que ofrece un mecanismo de justificación de las acciones de gobierno.

En términos más generales, debe señalarse que los diferentes autores que problematizan y presentan el proceso por etapas, sostienen que el ciclo de las políticas públicas no posee un orden preestablecido, pudiendo identificarse superposiciones entre las diferentes etapas, pero siguiendo un camino similar en el proceso. En resumen, las principales componentes del proceso de política pública son: temas que aparecen en la agenda; actores que presentan, interpretan y/o responden a esos temas; recursos afectados; instituciones o agencias que se ocupan de los temas; y los niveles del gobierno que dirigen los temas.

Otro aspecto importante señalado en relación con las políticas públicas es que suponen un proceso de aprendizaje en el cual participan diferentes actores que van construyendo intereses y preferencias de acuerdo a los incentivos creados por esfuerzos gubernamentales previos (Hall, 1993). En este enfoque las instituciones son consideradas como aquellas normas de juego que determinan las acciones humanas y estructuran incentivos en el intercambio político, social y económico; en definitiva las instituciones son importantes porque moldean las relaciones de poder pero también éstas se ven modificadas por las preferencias de los actores individuales.

Si entendemos, como lo hace Gerston, la política pública como la "combinación de decisiones básicas, compromisos y acciones llevadas a cabo por aquellos que detentan cargos o que influyen sobre los que poseen posiciones de autoridad" (Gerston 2010:7), veremos que en muchas ocasiones las acciones resultan de la interacción entre los actores que 
toman las decisiones y aquellos que son afectados por las políticas; y que este vínculo es parte constitutiva de cada una de las etapas del proceso mencionadas anteriormente.

\section{El proceso de implementación}

Pallares (1986), sintetiza tres grandes momentos del proceso de políticas públicas: el diseño de las políticas o su formulación, la implementación o su proceso de aplicación práctica y la evaluación de su rendimiento. Puede decirse que la implementación de una política pública es un tema de reciente interés, apenas en los años setenta algunos autores llamaron la atención sobre la necesidad de realizar estudios sobre la ejecución, dejando de lado la creencia tácita, inicialmente no problematizada, de que una vez que se tomaba la decisión esta sería implementada sin alteraciones ni modificaciones, logrando un resultado similar al inicialmente pensado.

Hasta finales de la década de 1960 la atención estaba puesta en lo que había que hacer y de qué manera. Lo que efectivamente se hacía cuando la decisión se ponía en marcha era potestad de los empleados gubernamentales, administradores o burócratas. Con el tiempo y a partir de los conflictos e inconvenientes que acontecían cuando la política efectivamente se ejecutaba, los analistas comenzaron a prestar atención a la implementación como parte de la decisión política y por lo tanto, como instancia vinculada al diseño.

Retomando la implementación como momento y precisando los enfoques desarrollados para los análisis mencionados anteriormente, Revuelta Vaquero (2007:139) la define como "el proceso que ocurre entre las declaraciones formales de la política y el resultado formal alcanzado". El autor analiza los diferentes modelos para la implementación de políticas públicas, en pos de la búsqueda de un enfoque que tenga en cuenta los marcos teóricos existentes y que pueda ser utilizado para abordar la implementación desde una perspectiva pluralista. ${ }^{24}$ El proceso de implementación puede ser verse afectado por una multiplicidad de variables y/o factores como el contexto, el tipo de política que se trate, la cantidad y disponibilidad de recursos, las condiciones socioeconómicas y la burocracia, entre otras. Para Revuelta Vaquero (2007) la burocracia se presenta como una de las variables más importantes, con capacidad de acompañar u obstaculizar el alcance de los objetivos previamente establecidos.

En un intento teórico y práctico complementario de esta mirada, Rain y Ravinovitz (1978) habían establecido tres imperativos de la implementación: el legal, el burocrático y el consensual. ${ }^{25}$ Estos autores sostienen que la implementación se trata de una declaración gubernamental mediada por diferentes actores que generan un proceso de negociación continua en el que se ponen en juego relaciones de poder. Desde esta perspectiva, los tres imperativos operan conjuntamente:

Por muy molesto que esto resulte en opinión de quienes prefieren que haya una división metódica del trabajo entre 
los políticos, los grupos de interés y los burócratas, debemos aceptar la realidad de que el proceso de implementación existe precisamente debido a que no es posible formular regla alguna de negociación con anterioridad a la práctica (Rain y Rabinowitz 1978:149).

Las tensiones entre los imperativos de la implementación se resuelven en función de los objetivos, los recursos y la complejidad del proceso administrativo. En este sentido, Rein y Rabinowitz (1978:178) explican que

Cuando hay propósitos claros, pertinentes y concretos, cuando las etapa de la implementación suponen la menor complejidad y cuando se cuenta con gran cantidad de recursos, se puede prever que los programas limitaran al mínimo posible la discrecionalidad y las desviaciones, con un amplio margen de consenso.

En términos operativos, los imperativos se desenvuelven en las denominadas etapas del proceso de implementación: la elaboración de lineamientos, la distribución de recursos y la supervisión. Estas tres etapas son interdependientes y revisten un carácter circular que debería ponerse a funcionar a partir de los resultados de la etapa de supervisión. Para que esto suceda es necesario que los resultados enunciados para un determinado programa sean lo suficientemente abarcativos, como para influir y producir cambios en el propósito y actividades del mismo. Los factores influyentes y variables determinantes del proceso de implementación mencionados anteriormente, afectan también a las etapas que lo componen.

\section{Algunas características de las políticas públicas en Ciencia y Tecnología}

Cuando el trabajo de los científicos se transformó en un medio para lograr los fines nacionales, la calidad de su actividad, la orientación y los medios necesarios para desarrollarla se convirtieron en materia de las políticas del gobierno. Surgió así la política científica contemporánea, como un campo particular en el conjunto de las políticas económicas (Albornoz, s/f).

Se define política científica como las políticas de un Estado en relación con la ciencia, es decir, aquellas destinadas a promover "el desarrollo de la investigación científica, el proceso de la innovación tecnológica, o el uso de la ciencia y la tecnología para objetivos políticos generales” (Moya, E. en Sanz Merino 2008:87).

Algunos autores como Sanz Merino (2008) afirman que la política científica y tecnológica se divide en tres subtipos fundamentales: política de promoción, política de regulación y política de evaluación. En América Latina y en Argentina, sostienen estos autores, la política de regulación -que se refiere al modo en que los entes gubernamentales asumen 
el rol de controlar efectos ambientales, sanitarios, sociales, económicos derivados de las actividades científicas- tiene poco desarrollo; los actores que participan de estas actividades son entes reguladores como la Administración Nacional de Medicamentos, Alimentos y Tecnología Médica (ANMAT) ${ }^{26}$ o los Comités de Ética de las diferentes instituciones. En cuanto a las políticas de promoción, señalan que incluyen tanto el diseño como la ejecución de las estrategias para fomentar desde el Estado las actividades de investigación en todas las disciplinas tales como: el incentivo por la vocación científica, el pago de becas; el apoyo para el financiamiento de centros de investigación tanto universitarios como dependientes de otros organismos públicos, los subsidios, los créditos y líneas destinadas a la innovación productiva en el sector privado. El último tipo de política a la que se refieren estos autores es la de evaluación de la ciencia y la tecnología, que puede incluir desde la evaluación ex-ante que se realiza para implementar estrategias de promoción, hasta la evaluación de impacto que se debe realizar al finalizar un programa o proyecto.

En la implementación de estrategias de promoción del sector científico y tecnológico la evaluación ex-ante es aquel proceso mediante el cual se decide la distribución de beneficios materiales o simbólicos por lo que reviste una importancia fundamental que afecta al proceso de implementación como tal. Son los procedimientos formales y prácticas llevadas a cabo por las entidades de promoción para decidir la adjudicación de algún beneficio, generalmente en el marco de las convocatorias públicas. La política de evaluación en ciencia y tecnología es puntualmente la forma por la cual se decide la distribución de gran parte de los recursos.

\section{Instrumentos de políticas de promoción de CTI en Argentina}

En nuestro país la ciencia y la tecnología alcanzan reconocimiento durante las décadas de 1950 y 1960. Ambas son recordadas ampliamente, por analistas e historiadores, como una "época de oro". ${ }^{27}$ Ya se señaló que a ese período corresponde la fundación de la mayoría de las instituciones de carácter público destinadas al diseño y la promoción de instrumentos para el desarrollo científico y tecnológico: la CNEA, en 1950; el INTI, en 1956; el INTA y el CONICET en 1957. De acuerdo a Albornoz (2008) la creación de estos organismos se debe en gran parte a tres factores específicos. El primero es el auge de las teorías del desarrollo en Argentina y América Latina durante el período 19501960. Estas teorías incluían, entre otras cuestiones, la preocupación por el impulso de la ciencia y la tecnología como camino para el desarrollo económico nacional y la consecuente mejora de las condiciones de vida de la población. El segundo factor es el consenso sobre el papel central del Estado en el estímulo a la producción de conocimientos científico-tecnológicos y el tercer factor que señala el autor es la consolidación de modelos institucionales para la promoción, planificación y producción de conocimiento. Posteriormente los países de América Latina comenzaron a homogenizar sus políticas científicas, a través de las directrices de organismos como la UNESCO y la OCDE, 
principales organizaciones de asesoría en política científica. La primera, centrada fundamentalmente sobre los países en desarrollo, y la segunda, de cara a los industrializados. (Albornoz,s/f). A éstas podría agregarse el Banco Interamericano de Desarrollo (BID), que si bien se incorpora a la escena de la política en ciencia y tecnología en Argentina en la década de 1960, irrumpe con todas sus fuerzas a partir de los años noventa. ${ }^{28}$

Así, las políticas de fomento a la innovación incorporaron los marcos teóricos que enfocan el proceso desde una perspectiva sistémica y se transformaron en políticas de estímulo al "Sistema Nacional de Innovación".

La irrupción de la noción de innovación tuvo en gran medida -al igual que otros enfoques provenientes de los países centrales- las características de un impulso mimético. Lo menos que puede decirse de la incorporación de esta idea, desde el enfoque del SNI, es que se trató de la aplicación no siempre crítica de modelos que interpretan realidades de otros contextos. A modo de ejemplo, puede mencionarse que a finales de 1996 el gobierno argentino comenzó la elaboración del Plan Nacional Plurianual 1999-2001, la primera línea de texto enuncia como objetivo "El desarrollo y fortalecimiento del Sistema Nacional de Ciencia, Tecnología e Innovación (en adelante Sistema Nacional de Innovación o SNI)".

Si bien son principalmente los políticos quienes deciden (López Cerezo 1996), es cierto que un grupo social -el de los expertos- juega un papel igual de importante, ya que tanto las decisiones como las modalidades que definen para llevarlas a cabo dependen en gran medida de sus consejos y advertencias.

En la exploración respecto de cómo y quiénes construyen la agenda sobre el sector de ciencia y tecnología, se observa que las estrategias de política pública de ciencia, tecnología e innovación las deciden, evalúan, implementan y están destinadas a un mismo y único actor: el científico. Esta característica, es una de las variables que afectan el proceso de implementación de las políticas de promoción.

En 2001 se sanciona la Ley $N^{0}$ 25.467, que constituye el marco legal del Sistema Nacional de Ciencia, Tecnología e Innovación en Argentina, el cual impulsa y promueve en su artículo primero:

Las actividades de Ciencia, Tecnología e Innovación, a fin de contribuir a incrementar el patrimonio cultural, educativo, social y económico de la Nación; propendiendo al bien común, al fortalecimiento de la identidad nacional, a la generación de trabajos y a la sustentabilidad del medio ambiente.

Como afirma Del Bello (2014), la ley cristaliza la reforma institucional establecida en 1996 garantizando la continuidad de organismos ya existentes y la asignación de 
funciones de formulación de políticas, medidas e instrumentos. En 2005, la Secretaría de Ciencia y Tecnología de la Nación (SECYT), en coordinación con el Observatorio de Ciencia, Tecnología e Innovación Productiva presentaron las Bases para un Plan Estratégico en Ciencia, Tecnología e Innovación 2005-2015. ${ }^{29}$ El documento fue considerado un trabajo fundamental cuyos aspectos principales serían retomados en posteriores documentos de política, en particular en la búsqueda de vínculos entre ciencia, tecnología y el sector productivo. De acuerdo a Porta y Lugones fue

...un primer intento de integrar las políticas en cuestión $y$ articularlas con el entramado productivo. Este documento constituyó un antecedente directo para la formulación del Plan Estratégico Nacional de Ciencia, Tecnología e Innovación "Bicentenario" (2006-2010). Las Bases contienen los lineamientos centrales para la política y la planificación de estas actividades entre 2005 y 2015 e incluyen una serie de metas cuantitativas y cualitativas, algunas de las cuales deberían ser alcanzadas en el bicentenario y otras dentro de los diez años previstos por las Bases (Lugones y Porta 2011:7).

El intento de elaborar un plan estratégico con metas concretas expresa, por un lado, el claro propósito de crecimiento y consolidación institucional, y por el otro la expectativa de alcanzar determinados niveles de desempeño del sistema nacional en función tanto de las necesidades del propio sistema, como del aporte del mismo al desarrollo nacional.

Algunas de las metas estratégicas establecidas para el año 2015 eran el aumento de la inversión total en I+D hasta llegar al 1\% del PBI, el incremento del financiamiento privado a la $\mathrm{I}+\mathrm{D}$ hasta equiparar el aporte público, el crecimiento del número de investigadores y tecnólogos hasta alcanzar el 3\%o de la PEA y la duplicación de la participación en el total de los recursos de I+D de las diecinueve provincias que concentraban sólo alrededor del 20\% de los mismos.

Para el cumplimiento de las metas mencionadas era necesario ampliar la batería de instrumentos de política en Ciencia, Tecnología e Innovación (CTI) disponibles. El aumento de los instrumentos de política de promoción de la CTI fue posible a partir de un programa financiado por el Banco Interamericano de Desarrollo (BID), a través de un préstamo con el Estado nacional. ${ }^{30} \mathrm{El}$ programa a desarrollarse durante el período 2006-2010 se denominó Programa de Modernización Tecnológica (PMT III).

A partir de la creación del Ministerio de Ciencia, Tecnología e Innovación Productiva en 2007, los instrumentos de política de promoción buscan profundizar la vinculación entre la industria, del sector productivo y el proceso de producción de conocimientos. 
Asimismo, el gobierno definió políticas de promoción en sectores de alta tecnología mediante incentivos fiscales específicos y herramientas de financiamiento. Tal como sostienen Abeledo y Del Bello (2007), las estrategias y herramientas de política pública en ciencia, tecnología e innovación refuerzan el cuerpo institucional construido diez años atrás a través de los fondos de la Agencia Nacional de Promoción Científica y Tecnológica.

La creación del MINCYT y la incorporación al discurso político de la noción de innovación como factor clave de la competitividad del país, fueron un claro signo de que la política para la ciencia, la tecnología y la innovación intentaba convertirse en Política de Estado.

\section{Implementación de instrumentos de promoción de la CTI}

La política científica se constituye en un sector específico de las políticas públicas. Siguiendo a Albornoz (s/f), el objetivo de la política científica consiste ofrecer a las actividades científicas los medios óptimos para su desarrollo y definir las posibles contribuciones del conocimiento científico al desarrollo nacional. Para dar cumplimiento al mismo, el sistema institucional de ciencia y tecnología debería desempeñar las funciones de Planificación, Coordinación, Promoción y Ejecución. Cuando hablamos de políticas públicas de promoción de la ciencia y la tecnología nos referimos a un fragmento puntual de la política científica, dedicado a "crear las condiciones necesarias para la realización de los objetivos, al otorgar recursos y verificar si su utilización produce los resultados esperados" (Albornoz s/f:49).

Zurbriggen y González Lago (2010) sostienen que las políticas de promoción en Argentina son de carácter horizontal, bajo un esquema orientado por la demanda (entendiendo, en el sentido señalado previamente por otros autores, como demanda de los expertos y no de los potenciales usuarios), en el cual a partir de la postulación de propuestas por parte de los beneficiarios potenciales y su consecuente evaluación, se canalizan los recursos hacia actividades de ciencia, tecnología e innovación. Las autoras se basan en el modelo identificado por la CEPAL de "esquema orientado por la demanda”, en el que se señala que las políticas del sector implementadas en América Latina, presentan

fallas frecuentes de Estado, tanto en lo que refiere a los procesos de evaluación de impactos como a la implementación en sí misma, producto de la fijación de metas no plausibles de ser concretadas por la debilidad en el diseño de los instrumentos para su ejecución (Zurbriggen y González Lago, 2010:18).

Estas fallas se centran en la necesidad de que el Estado reconozca que ya no corresponde un esquema de asignación de recursos impuesto en función de prioridades definidas políticamente, que representa una gran incertidumbre respecto de la implementación de 
una política pública. Sino en la necesidad de políticas públicas que den cuenta del carácter social, colectivo y contingente del proceso. Esta apreciación refuerza la idea de que la etapa de implementación forma parte constitutiva -en este caso- de la política científica. Asimismo, refleja el carácter cíclico de las políticas públicas poniendo en evidencia que las dificultades de la implementación deben formar parte de la formulación. La habilidad para reconocerlas, la capacidad para enfrentarlas y la pericia para preverlas, es lo que facilita el desenvolvimiento integral de las acciones de la política pública. La implementación de una política pública entendida como parte de la decisión política y como momento relacionado al diseño, ofrece la posibilidad desde su concepción analítica de observar las distancias entre aquello que se declara formalmente y el resultado final alcanzado.

Algunos autores como Franco y Lanzaro (2006) sostienen que las reformas del Estado posteriores a los años ochenta en América Latina representaron cambios para las políticas públicas; en ese marco, las investigaciones de la región se han centrado en la relación entre política y políticas públicas, a partir de casos de estudio respecto del análisis de la influencia de las variables políticas y de los actores como determinantes del proceso de elaboración e implementación de las políticas públicas. Asimismo, resaltan que los avances en el campo académico han sido significativos, pero que no han alcanzado la misma relevancia en la práctica de los programas.

Por su parte, Lahera (2005) sostiene que el peor déficit en América Latina no son las políticas en sí, sino la capacidad de implementación de políticas en general. El autor subraya que el déficit de la implementación no se encuentra determinado sólo por las capacidades preexistentes, sino también con la diferencia entre lo propuesto y lo alcanzado.

Retomando las definiciones de los autores mencionados en relación con el proceso de implementación y sus etapas, podemos sostener que durante las etapas de elaboración de lineamientos, distribución de recursos y supervisión se producen interacciones entre los actores que intervienen y forman parte de ese proceso. Asimismo sólo en el transcurso de esa interacción, es decir, durante la práctica, pueden reconocerse las pautas del proceso de negociación que los mismos instauran. Los niveles de articulación entre los actores del complejo ciencia, tecnología e innovación representan una cuestión crítica para su funcionamiento virtuoso, que ha sido resaltada en varios documentos citados a lo largo del trabajo. Particularmente desde la noción de "triángulo de Sábato" y la importancia que se atribuye a las instituciones encargadas de la política CTI en el diseño e implementación de actividades, conforme a las características que adquieren esa vinculación e interacción en cada región. 


\section{La implementación del Programa de áreas estratégicas (PAE 2006)}

En abril del 2006, el estado -a través de la ex Secretaría de Ciencia, Tecnología e Innovación Productiva (SECYT) - firmó con el Banco Interamericano de Desarrollo el contrato de préstamo BID N N $^{0}$ 1728/OC-AR para cooperar en la ejecución del Programa Modernización Tecnológica III (PMT III-8/2006). El programa comprometía 510 millones de dólares a ejecutarse entre el 2006 y el 2009; tenía como fin:

fortalecer la capacidad del país en ciencia y tecnología para dar respuesta a problemas sectoriales y sociales prioritarios $y$ contribuir a incrementar en forma sostenible la competitividad del sector productivo, sobre la base del desarrollo de un nuevo patrón de producción basado en bienes y servicios con mayor densidad tecnológica.

Su propósito era:

contribuir al fortalecimiento del Sistema Nacional de Innovación y de los Sistemas Regionales de Innovación, aumentando las capacidades innovativas $y$ de asociatividad de sus actores, contribuyendo al desarrollo de una infraestructura científica y tecnológica moderna que apoye los esfuerzos nacionales orientados a generar conocimientos, y a integrar la CyT a las diversas actividades y sectores de la sociedad argentina. ${ }^{31}$

El estudio del Programa de Áreas Estratégicas (PAE) se realizó a partir de un abordaje mixto con foco en el estudio de su dinámica como instrumento asociativo de promoción CTI. La información recolectada proviene de la selección de documentos de carácter público existentes sobre el instrumento y del análisis de entrevistas. El punto de partida lo constituyen las fases que el instrumento atraviesa durante la implementación. Así, para las fases de formulación y evaluación se realizó el análisis de información (documentos normativos y regulatorios, de evaluación, información de gestión disponible en el organismo público encargado de la implementación) que establece los lineamientos y dan origen al instrumento PAE. Para las fases de ejecución y seguimiento se realizó el análisis documental de los mecanismos formales construidos para la medición de resultados y entrevistas a los actores beneficiarios de algunos casos seleccionados y al personal técnico del organismo ejecutor.

El análisis documental y las entrevistas se llevaron adelante en base a tres ejes:

- La producción de conocimiento a través de la interacción de actores.

- La Gestación del instrumento como cluster, proyecto integrado y asociativo. 
- El correlato de la integración y la interacción de actores durante las etapas de la implementación.

Es importante señalar que el programa se basaba en un diagnóstico favorable de la situación económica del país, lo que implica de alguna manera una visión de oportunidad y una orientación tácita. Tal como lo expresa un informe publicado en 2010, "El Programa de Modernización Tecnológica III (PMT III) se diseñó y aprobó en un contexto de recuperación económica, posterior a la profunda crisis que sufrió la Argentina en 2001-2002, en la que se registró una pérdida del $20 \%$ de su producto bruto interno (PBI)". ${ }^{32}$

Ese mismo año, la Secretaria de Ciencia y Tecnología (SECYT) publicó el Plan Estratégico Nacional de Ciencia, Tecnología e Innovación “Bicentenario” (2006-2010). El Plan Bicentenario se autodefinía como un "proceso continuo e interactivo de definición de estrategias, objetivos y metas" y dejaba roles claramente establecidos en el ámbito de las políticas públicas de ciencia, tecnología e innovación nacionales. Así, la SECYT figura como el organismo responsable de elaborar y proponer políticas por medio del Plan y evaluar su desarrollo y cumplimiento; y la Agencia Nacional de Promoción Científica y Tecnológica (ANPCYT) como responsable de la promoción de las actividades. Esta última debía gestionar y adjudicar fondos "a través de evaluaciones, concursos, licitaciones o mecanismos equivalentes que garanticen transparencia”.

Entre las metas proyectadas en el plan se encuentra el incremento proporcional en la inversión en ciencia y tecnología por parte del sector privado ${ }^{33}$ y la hipótesis de que el mismo alcanzaría hacia 2010 el 50\% de la inversión nacional total en ciencia y tecnología. ${ }^{34}$ Asimismo, el Plan presenta un apartado en el que refuerza el aumento de inversión en el sector con la coordinación de políticas públicas en relación con un Sistema Nacional de Innovación.

Bajo esta idea de coordinación de políticas y constitución de un SNI integrado, el Plan Bicentenario presentaba una serie de programas, acciones e instrumentos entre los que se encontraba el Programa de Áreas Estratégicas (PAE), ${ }^{35}$ cuyo objetivo era la conformación de "clúster de conocimientos", y el apoyo a la realización de proyectos integrados en áreas y temas prioritarios establecidos previamente en el mencionado plan. ${ }^{36}$

A diferencia de otras líneas de financiamiento de la ANPCYT, el PAE pretendía, a través de la interacción sinérgica de las instituciones involucradas, su carácter (público y privado), y sus diferentes funciones en la producción de conocimientos, la mejora de la competitividad del sector productivo y el desarrollo social. Para la conformación de los clústers, el PAE debía estructurarse sobre un conjunto de subproyectos financiables con los instrumentos regulares de los fondos pertenecientes a ANPCYT (FONCYT y FONTAR). Los instrumentos de estos fondos, que existen como líneas de financiamiento independientes de la Agencia, presentan características diversas en cuanto a 
actores participantes, montos a financiar, requisitos de presentación, tiempos de ejecución, tipo de objetivos y resultados esperados. El nuevo instrumento debía reunir estas particularidades en su formulación.

Si pensamos en la etapa de elaboración de lineamientos dentro de la implementación del PAE como el momento de apertura de la convocatoria, podemos identificar la coexistencia de diferentes modelos de gestión. En un primer momento, surgen rasgos del modelo top down, donde la decisión política viene dada en el contrato del préstamo y el reglamento operativo, mientras que la burocracia personificada en los cuadros de la administración pública establecidos por la misma decisión política, la ejecuta sin alterar el curso ni las reglas establecidas en la instancia superior. Por otra parte si nos posicionamos sobre las bases de la convocatoria como el punto de partida para la asignación de recursos y las analizamos en clave del modelo de interacción podemos afirmar que la implementación en su etapa de elaboración de lineamientos presentó a partir de la definición de las reglas del instrumento la raíz de las dificultades que debió asumir el proceso de implementación del PAE debido a su complejidad. Entre ellas se destacan los requisitos de: agrupar actividades de naturaleza y finalidades distintas; la participación de actores con diferentes intereses, objetivos y funciones; el agrupamiento de componentes con reglas de funcionamiento y mecanismos de evaluación diferentes y la diversidad de organizaciones participantes.

El obstáculo más difícil de sortear quizás haya sido la necesidad de combinar instrumentos que debían desenvolverse de forma independiente y bajo reglas propias. El hecho de que el PAE haya establecido como requisito la articulación de un conjunto de instrumentos preexistentes es una variable que refuerza los inconvenientes mencionados, desalentando la asociatividad de los grupos de trabajo. Al ser un instrumento compuesto por otros, con años de trayectorias individuales y que han desarrollado tiempos y lógicas de funcionamiento diferentes, la posibilidad de trazar un plan de trabajo conjunto se vio entorpecida, acentuando la tendencia de la formación de subgrupos sin conexión entre sí.

Como instrumento de promoción, el PAE fue la primera experiencia de puesta en marcha de un programa desde el ámbito de las políticas públicas de promoción CTI, con un importante nivel de complejidad en el financiamiento y tiempo de ejecución; así como en la pretensión de articulación de sectores e institucionalización de proyectos. Las limitantes del proceso de la implementación mantienen relación con la complejidad de la acción conjunta, evidenciada por la multiplicidad de actores que intervienen en el proceso y la puesta en juego de sus intereses; así como las características que la burocracia adoptó y los tipos de organizaciones involucradas.

Las etapas de elaboración de lineamientos y distribución de recursos se identifican en el PAE con las acciones de formulación y evaluación que la ANPCYT debió llevar adelante. En el transcurso de esas acciones se producen interacciones, entre la burocracia 
y los beneficiarios. En este sentido, el instrumento PAE, parte integrante de la política que pretendía inaugurar el plan bicentenario, no recoge durante su implementación los supuestos de los que parte -integración, asociatividad, interacción- a pesar de poseer varios de sus atributos, que se constituyen como características -de existencia previa- en el conjunto de actores participantes del instrumento.

En términos de gestión de las etapas de formulación y evaluación durante la implementación, en los documentos rectores del proceso se identifican elementos relacionados con la naturaleza de las actividades, la heterogeneidad y diversidad de actores, instituciones y herramientas involucradas en los proyectos. La explicitación de estos elementos permite inferir que la formulación y evaluación del PAE, incluiría mecanismos consecuentes con un modelo de gestión interactivo.

La falta de herramientas que permitan evaluar el desempeño de los consorcios o de sus potenciales integrantes, para incrementar la interacción y evitar desvíos durante la formulación; junto a la ausencia de instancias de evaluación complementarias a los mecanismos tradicionales del control de calidad, significaron la reducción de la gestión del instrumento en un modelo en el que la burocracia llevó adelante las acciones planificadas sin posibilidad de flexibilización durante el curso de acción preestablecido.

En función de lo expuesto se identifican dos aspectos en relación con la gestión de proyectos integrados y complejos: la necesidad de introducción, durante la formulación y evaluación de los mismos, de mecanismos formalizados de interacción entre la burocracia y los beneficiarios en pos de la recolección y generación de datos que permitan el ajuste del instrumento; y la reorientación de acciones en pos del cumplimiento de los objetivos del instrumento diseñado.

La etapa de distribución de recursos comporta, en el marco del PAE, las fases de evaluación y ejecución. Del análisis de la fase de evaluación surgen interrogantes en relación con el desfase y/o desvíos respecto del plan previsto y las medidas correctivas desde la gestión. Por otra parte, la respuesta a estas cuestiones arroja luz respecto de la etapa de supervisión, identificada con la fase de seguimiento de los proyectos. En términos de la gestión de los proyectos y su administración, el modelo identificado es aquel concordante con las prácticas establecidas por la estructura burocrático-administrativa que sostenían la ejecución de recursos y que presenta, al igual que en las fases anteriores, el derrame de decisiones y cursos de acción establecidos en una instancia superior. Asimismo, se evidencia la ausencia de interacciones concretas y formales entre los beneficiarios del programa y los encargados de su gestión, que permitan vislumbrar la posibilidad de ajuste sobre la marcha.

Desde el lado de los beneficiarios, la ejecución y el seguimiento es vista como ad hoc, con procesos de gestión personalizados y bastante improvisados; y desde la perspectiva 
de los actores burocráticos la ejecución y el seguimiento se solapan con la marcha financiera y los montos erogados. La base burocrática de la gestión de los proyectos tanto administrativa como financiera no induce la integración, ni ofrece recursos humanos en cantidad y calidad necesaria para atenderlos de manera satisfactoria. Esto se observa en la fase de seguimiento, en la que se supone que las tareas de supervisión de un proyecto estratégico incluyan desde la gestión mecanismos tendientes al cumplimiento de las condiciones de unicidad y el carácter integrado.

\section{Corolarios y recomendaciones: La producción de conocimiento en contexto y los sistemas nacionales}

Las nociones pertenecientes a determinados marcos conceptuales incorporadas en el discurso de la política CTI impregna el discurso de los instrumentos que a través de éstas se diseñan dejando marcas e implicancias en el proceso de implementación.

En este sentido la incorporación de conceptos, particularmente el de Sistema Nacional de Innovación (SNI) es parte de un proceso situado a mediados de los '90 a partir de los cambios institucionales que significaron la reforma del Estado. La noción de SNI ha sido representativa de este proceso desde su incorporación en los documentos de organismos internacionales de crédito como el Banco Mundial (BID) y el Banco Interamericano de Desarrollo (BIRF), constituyéndose en el basamento para el diseño de políticas públicas de desarrollo. En este caso particular el concepto ha funcionado como articulador de un discurso que da lugar al instrumento PAE y como noción que lo atraviesa inclusive durante el proceso de implementación.

En el momento en que la implementación se convierte en una actividad meramente técnico-administrativa las marcas se desdibujan y la gestión no se pregunta ni cuestiona de dónde vienen los conceptos, si son aplicables o apropiables a nuestras condiciones de producción de CTI, a nuestros contextos locales. Así la implementación pareciera dejar de formar parte de ese proceso de la política adquiriendo vida propia.

Este mismo proceso a partir del cual emerge la noción de SNI, sumergió otras y con ello las implicancias de un Sistema Nacional de Ciencia y Tecnología situado local/ regionalmente. Conjuntamente implicó el abandono de la perspectiva crítica hacia la implantación de modelos heredados y la orientación de políticas instauradas por el Pensamiento Latinoamericano en Ciencia, Tecnología y Sociedad (PLACTS). Como parte del mismo proceso, se legitimaron e ingresaron a la agenda CTI políticas, programas e instrumentos que incentivan la asociatividad y la producción de conocimiento para la resolución de problemas, con miras a lograr el funcionamiento sistémico. 
Por otra parte, los cambios en el diseño de las acciones de promoción, que forman parte de la política pública en CTI, necesariamente suponen consecuencias a la hora de la implementación y en esa etapa se evidencian los preceptos para su institucionalidad.

Las conceptualizaciones en relación al sistema nacional de innovación pasaron a formar parte del discurso, tanto de los nuevos planes y programas como de los documentos de los organismos que aportan recursos al sector.

Así, los marcos referenciales establecidos ingresan a las políticas del sector dando lugar, en este caso, a instrumentos que promueven la creación de redes, aglomerados productivos, clústeres de conocimiento con base en la articulación público-privada y la implicación e incorporación de nuevos actores.

En una época marcada por la fuerte tendencia al aumento en el gasto en ciencia, tecnología e innovación la creación de instrumentos destinados a estimular y fortalecer el SNI debió estar acompañada de un proceso de aprendizaje institucional que facilite desde el proceso de implementación el funcionamiento sistémico. El carácter transitorio de las acciones conjuntas para la resolución de problemas, junto a las pocas muestras de continuidad de las mismas, permiten cuestionar, cuando no poner en dudas, el aporte genuino de los instrumentos diseñados en el estímulo y fortalecimiento del carácter sistémico a nivel local.

En este sentido, los análisis de la implementación de políticas se constituyen en el termómetro de las condiciones de posibilidad de una acción determinada y el grado de complejidad desde la perspectiva de la multiplicidad de actores y factores que intervienen. Por otra parte, es importante que se incorpore al análisis de los procesos de implementación una mirada pluralista que recoja e interrelacione los diferentes enfoques y marcos conceptuales producidos. La utilidad de los enfoques radica en el aporte que estos realizan para la explicación de un fenómeno, pero muchas veces nos colocan en una perspectiva de análisis que nos impide pensar en su convivencia. Sin embargo, esta convivencia permite la generación de información relevante e inicia el camino hacia nuevas formas de abordaje, siempre y cuando la realidad del fenómeno otorgue algún asidero. En cualquier caso, la utilización e interpretación de enfoques aplicados a otros contextos debe, al menos, analizarse desde el posicionamiento de una actitud crítica.

Acortar esta brecha iniciaría el camino hacia lo que se define como una política pública de excelencia, que plantea objetivos claros y logra definir de la mejor manera temas técnicos. Si bien estos últimos no pueden reemplazar la política, la complementan para darle forma y en este sentido forman parte de ella. El proceso de mejora debe ser selectivo y debe poder determinar qué es lo que se quiere mejorar. Asimismo, para la mejora de las capacidades de implementación es necesario entender al sector público, no como factor ajeno a cómo se deciden e implementan las políticas, sino como endógeno al proceso. 
Si bien no existen recetas generales para el cambio institucional necesario para que las políticas CTI, que formaron parte de la reforma del Estado, se conviertan en políticas de excelencia, los estudios de los procesos de implementación abren una puerta para lograr una mejor articulación entre las consideraciones políticas y las técnicas.

Por último, si un Sistema Nacional de Innovación es un proceso de aprendizaje -del cual el desarrollo tecnológico es parte- acumulativo, incierto y arraigado en las relaciones entre organizaciones e integrado no solo a las actividades desarrolladas por las instituciones de ciencia y tecnología, su construcción necesariamente es histórica y las interacciones surgen de sus condiciones de posibilidad.

El diseño de un instrumento de política debería, de mínima, tener en cuenta las condiciones de posibilidad, e incorporar en las áreas de planificación y gestión el análisis en relación con el enfoque conceptual adoptado, su contribución, y las posibles distorsiones que puede introducir en la apreciación del contexto en el que se pretende aplicar. La crítica del PLACTS a las orientaciones de política propuestas por UNESCO en los años 50, presuntamente desinteresadas, se vuelve vigente, poniendo en evidencia que cada proceso de aprendizaje por interacción debería ser útil para delinear políticas que efectivamente respondan a necesidades locales y/o regionales, y consecuentemente deberían permitir una gestión de instrumentos que no se reduzca a la actividad de los actores aguas abajo de la decisión política y bajo el paragua de marcos conceptuales importados. Se trataría entonces de un proceso de aprendizaje retroalimentado con el aporte de categorías locales que permitan el abordaje de nuestra realidad como país.

Si la comprensión de nuestro complejo de ciencia, tecnología e innovación, o de nuestro sistema actual de ciencia, tecnología e innovación, le diera uso a la noción de sistema para identificar espacios regionales y locales donde las actividades relacionadas con la ciencia, la tecnología y la innovación se congregan, y si sostuviéramos que en el mismo se producen interacciones diferentes entre actores del sector productivo, científico y gubernamentales, que se movilizan conforme a sus intereses, contextos e instituciones culturales, educativas, etc., en la producción de conocimiento, entonces, la política y la gestión deberían tomar este marco como el lugar donde se produce el aprendizaje, donde la innovación en última instancia también sería una construcción contextual y algo diferente en cada caso.

A la luz de las conclusiones vertidas sería deseable la formulación de un cuerpo teórico y metodológico que permita relevar los resultados de la implementación, la creación de herramientas para facilitar la interacción a lo largo de toda la cadena institucional de la cual forma parte la implementación; el diseño de herramientas administrativas y de gestión que permitan el ajuste de los instrumentos diseñados sobre la marcha; junto a la consecuente capacitación de los cuadros burocráticos y técnicos específicos para el proceso de implementación. 
Desde una perspectiva en la que los cursos de acción posteriores a la formulación de instrumentos de política, son parte de la política, los análisis de procesos de implementación orientados al estudio de la puesta en marcha de instrumentos política pública CTI, pueden aportar información relevante para la gestión y poner en valor el estudio de caso como estrategia de investigación.

La información recogida durante el proceso de implementación de un instrumento de política puede resultar útil como insumo para la medición, entre otras cosas, del impacto, de la articulación de sectores y de los vínculos establecidos por las instituciones y actores participantes. La falta de herramientas que permitan evaluar el desempeño de un instrumento particular y de los actores participantes, junto a la ausencia de instancias de evaluación complementarias a los mecanismos tradicionales de control, se traducen en una gestión configurada sobre la rigidez de los mecanismos de administración del organismo designado para su implementación y en un trabajo de gestión que solo se ajusta a llevar adelante acciones generales, sin posibilidad de flexibilización durante el curso de acción preestablecido.

La implementación de políticas específicas que incorporan el marco conceptual de Sistema Nacional de Innovación y las características de una nueva forma de producción cognitiva y los instrumentos diseñados para tal fin, aportaron a la consolidación de una nueva institucionalidad pública para promover la investigación científico-tecnológica y la innovación, con capacidad para reproducir acciones durante cierto tiempo, que es lo que convierte a las acciones de gobierno en política pública. Sin embargo, el diseño de instrumentos de promoción para impulsar el funcionamiento sistémico, resultan insuficientes si se carece de una estructura de gestión para ello.

Las instancias de aprendizaje institucional contextualizadas en el proceso y las prácticas de producción de conocimiento que cada instrumento propone resultan claves en este punto, así como el ajuste en el proceso de gestión de los instrumentos significaría dos cuestiones fundamentales. Por un lado, la necesidad de introducir, durante las etapas de implementación, mecanismos formalizados de interacción entre los actores participantes, en pos de la recolección y generación de datos que permitan el acople del instrumento y la reorientación de acciones. Por el otro, la adopción desde las instancias burocráticas de una actitud proactiva hacia aquello que los burócratas hacen. De este modo, en el marco del proceso de una política pública, se lograría completar el mapa de la implementación con la información producida durante sus etapas, a la vez que incorporarla a los mecanismos establecidos para la evaluación de gestión. Así, se generaría un proceso de aprendizaje para la administración pública con una perspectiva de largo plazo, planeamiento estratégico y previsibilidad, dando muestras de fortaleza institucional. 


\begin{abstract}
${ }^{1}$ Los modelos de gestión describen la implementación como una de las etapas del proceso por el que atraviesa cualquier política pública.Vid. Aguilar Villanueva (1992) y Estevez et al (2002).

${ }^{2}$ Revuelta Vaquero (2007) menciona a Pressman y Wildavsky, Sabatier y a Van Meter y Van Horn, entre otros, como los principales autores que fortalecieron -principalmente en países desarrollados- la implementación como área de estudio que abarca acciones de individuos o grupos públicos y privados en busca del cumplimiento de los objetivos previamente decididos.

${ }^{3}$ Zurbriggen y González Lago (2010) mencionan el surgimiento de una nueva literatura relacionada con la producción de conocimiento y el proceso de innovación, producida desde la teoría del management, la economía de la innovación, la competitividad y la ciencia política que enfatiza cada vez más en el papel de las instituciones, las relaciones sociales etc., como elementos centrales para la competitividad y el desarrollo. ${ }^{4}$ Las principales fuentes de financiamiento externo para el sector científico y tecnológico ha sido el Banco Interamericano de Desarrollo (BID) desde la década de 1970 con una perspectiva de oferta que evolucionó hasta incorporar la noción de Sistema Nacional de Investigaciones como marco conceptual y metodológico. Más recientemente se incorpora con el mismo enfoque el Banco Internacional de Reconstrucción y
\end{abstract} Fomento (BIRF). Para un análisis completo ver Abeledo (2000).

${ }^{5}$ En 2008, las autoridades de los organismos de Ciencia, Tecnología e innovación de 8 países, entre ellos la Argentina, firmaron una declaración Ministerial afirmando la voluntad política de avanzar hacia la construcción de un mecanismo permanente para el diálogo entre políticas, y designan a la CEPAL como el encargado de coordinar las actividades y dirigir el proceso. (OCDE 2011).

${ }^{6}$ Los autores García Palacios, González, Galbarte, Lopez Cerezo, Lujan Gordillo, Osorio y Valdez (2001), distinguen entre la expresión "CTS" y "estudios CTS" para hacer referencia a las relaciones entre ciencia, tecnología y sociedad como objeto de estudio en el primer caso y para identificar el ámbito académico que involucra los enfoques recientes del estudio de la ciencia y la tecnología en el segundo.

${ }^{7}$ En la misma línea se destacan, entre otros, autores Ziman, J. (1986) y Woolgar, S. (1991).

${ }^{8}$ En este caso, el punto de partida para la gestación del campo CTS tenía que ver más con una crítica al formato de política en ciencia y tecnología contraria a las necesidades de desarrollo de la región, que con una crítica a las consecuencias del complejo ciencia y tecnología. (Vaccarezza 1998:13-40).

${ }^{9}$ Las contribuciones de Sábato $(1980,1982)$ en torno a la naturaleza de la tecnología y su participación en el proceso productivo, las fábricas de tecnología y el "triángulo" de interrelaciones; las de Herrera (1973) en relación con las vinculaciones entre el ámbito científico tecnológico y la cultura, los impactos socioeconómicos y los mecanismos de desarrollo y, la perspectiva de Varsavsky $(1969,1971)$, respecto de las conciencias públicas respecto de las tecnologías en relación a contextos específicos, forman parte, entre otros estudios, del PLACTS.

${ }^{10}$ Cuando hablamos de campo CTS nos referimos al espacio interdisciplinario en el que convergen estudios de la investigación académica, la educación y la política pública que pretenden dar cuenta de los aspectos sociales de la ciencia y la tecnología, tanto en relación a sus condicionantes sociales como, a sus consecuencias sociales y ambientales.

${ }^{11}$ Lundvall (2002) sostiene que el análisis en otros niveles es no sólo legítimo sino también necesario en función de otorgar una explicación realista del funcionamiento del sistema nacional, además de proveer una visión más aclarada de los límites y eficiencia de las políticas a nivel nacional.

${ }^{12}$ En el año 2010, el Ministerio de Ciencia, Tecnología e Innovación Productiva, creó en el ámbito de la Secretaría de Planeamiento y Políticas, el Programa de Estudios sobre el Pensamiento Latinoamericano en Ciencia, Tecnología y Desarrollo (PLACTED). Entre sus objetivos se propone "Analizar los desafios actuales del sector CTI a la luz del Pensamiento Latinoamericano como insumo estratégico para la definición de políticas públicas que promuevan la autonomía científica, tecnológica e innovativa”.

(http://www.mincyt.gob.ar/programa/placted-programa-de-estudios-sobre-el-pensamientolatinoamericano-en-ciencia-tecnologia-y-desarrollo-6414).

${ }^{13}$ Para profundizar respecto de las características del "modelo lineal de innovación” como orientación de instrumentos de política científica y tecnológica, denominada políticas de oferta luego de la Segunda Guerra Mundial, ver Albornoz (s/f).

${ }^{14} \mathrm{El}$ documento, denominado "Science and the Policies of Governments" fue publicado por la OCDE en 1963. (citado en Albornoz, 2007).

${ }^{15}$ Este nuevo documento fue publicado por la OCDE en 1981 y se tituló "Science and Technology Policy for the 1980s". (citado en Albornoz, 2007).

${ }^{16} \mathrm{El}$ artículo "El pensamiento en ciencia, tecnología y sociedad en Latinoamérica: una interpretación política de su trayectoria", realiza un interesante análisis en esta línea. Por su parte, Nuñez Rover (1999), aporta la perspectiva de la ciencia y la tecnología en relación al desarrollo social y plantea que la sustitución 
de la política científica y tecnológica por la política para la innovación, supone "el abandono de la pretensión de un desarrollo científico y tecnológico endógeno, relativamente autónomo”, y postula un tipo de política desvinculada de la sociedad real.

${ }^{17}$ Miró (s/f) realiza un análisis de caso desde la perspectiva relacional, e identifica como coordenadas teóricas dos enfoques. Uno de esos enfoques, centra su mirada en lo que sucede en la sociedad, por lo tanto las políticas son el resultado de los acuerdos y conflictos entre diferentes grupos sociales y, serán estos quienes moldearán los productos estatales. Otro de los enfoques cambia el eje desde las explicaciones centradas en acuerdos y conflictos entre diferentes grupos sociales hacia el concepto de Estado. Desde este enfoque se entiende que las políticas están hechas por agentes estatales que actúan de manera autónoma de las presiones de los grupos sociales.

${ }^{18} \mathrm{Si}$ bien el surgimiento de los primeros trabajos se le atribuyen a Laswell en la década del '50, a partir de los años posteriores que aparecen modelos sobre la elaboración de políticas. (Pallares 1988:141-162).

${ }^{19}$ Laswell $(1956,1971)$, es el primero en aportar un esquema denominado de "ciclo vital" para la interpretación del proceso de política pública. Pallares (1988) sostiene que el esquema de 'ciclo vital' de Lasswell se ha mantenido como punto de referencia de las diferentes adaptaciones posteriores.

${ }^{20}$ Los dos modelos han servido para representar los 'límites del camino' en la búsqueda de un modelo descriptivo y prescriptivo al mismo tiempo, intentando evitar tanto el idealismo subyacente en el modelo racional como la limitación de perspectivas inherente al modelo incremental. (Pallares 1988).

${ }^{21}$ Estévez (2009:72-90.) realiza un análisis del denominado Modelo Secuencial de políticas públicas surgido en EE.UU. en las décadas del 60/70, a partir de las influencias de la teoría sistémica aportada por Easton, D., los estudios de Simón. y Lasswell, entre otros.

22 Oszlak y O’Donnell, denominan a los asuntos problematizados socialmente, “cuestiones" y plantean que, todas atraviesan un "ciclo vital" que va desde su problematización hasta su resolución; pero no todos los asuntos tienen la posibilidad de convertirse en cuestiones, teniendo esto que ver con la interacción de actores y con su carácter contextual. (Oszlak y O’Donnell 1994:110).

${ }^{23}$ En este punto, puede observarse que los modelos "racional" e "incremental" -surgidos en los años '50 y '60- anteriormente mencionados, tenían como objetivo el abordaje de una de las etapas de las políticas públicas, focalizando los estudios en la etapa de formulación.

${ }^{24}$ La utilización de estos modelos ha sido foco de debate entre los analistas teóricos. Los interrogantes centrales son: una vez identificada y seleccionada la o las alternativas, ¿`cuál es el enfoque adecuado para poner en marcha la decisión? ¿Existe un solo modelo a adoptar para la implementación de políticas públicas?

${ }^{25}$ Los autores esperan que estos factores puedan ser considerados para la formulación de nuevas políticas, utilizan para la dar cuenta de la relación entre los imperativos que describen, ejemplos como el de la CNEA; poniendo especial atención a los actores que dominan el proceso de implementación, en este caso el actor científico.

${ }^{26}$ La ANMAT es un organismo descentralizado de la Administración Pública Nacional que depende técnica y científicamente de las normas y directivas que le imparte la Secretaria de Políticas, Regulación e Institutos del Ministerio de Salud. Se ocupa del registro, fiscalización, normalización y vigilancia de la sanidad y calidad de los productos, substancias, elementos, procesos, tecnologías y materiales que se consumen o utilizan en la medicina, alimentación y cosmética humana y del control de las actividades y procesos que están comprendidos en estas materias.

${ }^{27}$ La denominación de "época de oro" es utilizada por Albornoz (2008:187).

${ }^{28}$ Con el apoyo de créditos del Banco Interamericano de Desarrollo (BID), después de 1976, el gobierno militar de la Argentina instaura una política científica y tecnológica que focalizaba en el desarrollo institucional. En ese marco se produjo la creación de los Centros Regionales del CONICET. Para un análisis pormenorizado de la relación entre la evolución del pensamiento del BID y la evolución de los instrumentos de apoyo a la innovación, ver: Rivas y Rovira (2014). El documento sostiene que el BID fue la principal fuente de financiamiento de la reforma institucional, contribuyó a consolidar el CONICET -a través de los préstamos BID I y BID II-, y con ello el enfoque lineal ofertista. Luego el banco cambió su enfoque, hasta asumir el Sistema Nacional de Innovación como marco teórico y metodológico.

${ }^{29}$ El primer Plan Nacional de Ciencia y Tecnología (1971-1975) fue elaborado en 1971 y entre sus objetivos establecía “pasar del 0,27\% de inversión en ciencia y tecnología respecto del PBI en 1970, al $0,64 \%$ en 1975 y al mítico $1 \%$ en 1980 , meta aún no alcanzada y que ha figurado hasta ahora en todos los planes de ciencia y tecnología”. (Del Bello 2014:38).

${ }^{30}$ El Banco (BID) presta su ayuda a través de estudios de preinversión, asistencia técnica reembolsable o no reembolsable y operaciones de préstamo. Tiene como premisa procurar que la ayuda sea de carácter reembolsable, siempre que sea factible. Los proyectos de ciencia y tecnología pueden ser financiados con recursos del Fondo para Operaciones Especiales o del Capital Ordinario de acuerdo con las actuales pautas para el uso de estos fondos y según la naturaleza del proyecto y del país prestatario. El Banco apoya la 
formulación y aplicación de la política científica y tecnológica de los países miembros, que tenga como uno de sus propósitos integrar el desarrollo científico y tecnológico en los planes y políticas nacionales de desarrollo en general. (http://www.iadb.org/es/acerca-del-bid/politica-de-ciencia-y-tecnologia-del-bancointeramericano-de-desarrollo,6225.html).

${ }^{31}$ MINCYT, ANPCYT, Anexo Único contrato préstamo BID No 1728/OC-AR, 2006, p. 1. Disponible en: http://www.agencia.mincyt.gob.ar/archivo/1376/agencia/contrato-prestamo-bid-1728-oc-ar.

${ }^{32}$ BID, Informe de Terminación de Proyecto PCR 2010 P. 3, disponible en: http://idbdocs.iadb.org/ wsdocs / getdocument.aspx?docnum $=35593999$.

${ }^{33}$ El Plan Bicentenario establece esta meta en base a resultados obtenidos en la "Encuesta Nacional a Empresas sobre Innovación, I+D y TIC's (2002-2004)" publicada en octubre de 2006, realizada por el INDEC con datos de los años 2002 a 2004 y a una creciente dinámica empresarial apoyada por instrumentos públicos de promoción de la I+D y de la innovación. (Plan Estratégico Nacional de Ciencia, Tecnología e Innovación "Bicentenario” (2006-2010), Capítulo VIII).

${ }^{34}$ Desde 2003, se produjo una fuerte recuperación de la inversión pública en actividades científicas y tecnológicas (AC\&T). En aquel año, el presupuesto dedicado a AC\&T fue de US\$828 millones, mientras que en 2010 llegó a US\$5270 (aproximadamente US\$1.320 millones). (BID, 2010:3). Asimismo, la inversión en AC\&T creció más que el presupuesto nacional en su conjunto, aumentando su participación desde $1.38 \%$ en 2003 a 1,91\% en 2010. Con respecto al PBI, la inversión en AC\&T pasó de representar un $0,46 \%$ en 2003 a un $0,70 \%$ en 2010 (según los Indicadores publicados en 2011, disponible en http:// indicadorescti.mincyt.gob.ar/documentos/indicadores_2011.pdf, p.31).

${ }^{35} \mathrm{Al}$ momento de surgimiento del PAE conformaban la ANPCyT, el Fondo Tecnológico Argentino (FONTAR), el Fondo para la investigación Científica y Tecnológica (FONCyT) y el Fondo Fiduciario de Promoción de la Industria del Software (FONSOFT).

${ }^{36}$ Anexo Único contrato préstamo BID Nº 1728/OC-AR, pág.14. 


\title{
Referencias bibliográficas
}

Aguilar Villanueva, Luis (1992) La implementación de las políticas públicas. México: Porrúa.

\begin{abstract}
Albornoz, Mario (2001) "Política Científica y Tecnológica: una Visión desde América Latina”, Revista Iberoamericana de Ciencia, Tecnología, Sociedad e Innovación CTS + I, 1 . http://www.oei.es/revistactsi/numero1/albornoz.htm accesado el 1 de agosto 2014.
\end{abstract}

Albornoz, Mario (2007) "Los problemas de la ciencia y el poder", Revista Iberoamericana de Ciencia, Tecnología y Sociedad,V.3, No8. http://www.scielo.org.ar/scielo.php?script=sci_ arttext\&pid=S1850-00132007000100005\#notas accesado el 24 de mayo 2018.

Albornoz, Mario (2008) Claves del Desarrollo Científico y Tecnológico de América Latina. Jesús Sebastián (ed.), México: Siglo XXI.

Albornoz, Mario y Ariel Gordon (2011) "La política de ciencia y tecnología en Argentina desde la recuperación de la democracia (1983-2009)", en Albornoz M. y Sebastian, J. (eds) Trayectorias de las políticas cientificas y universitarias de Argentina y España, Madrid: CSIC, 2011:1-46.

Carrizo, Erica y Victoria Alfonso (2013) "Las políticas de CyT y el "estilo de desarrollo": un proyecto inconcluso", Voces en el Fénix, N²4:96-106.

Dagnino, Renato, Hernán Thomas y Amilcar Davyt (1996) "El pensamiento en ciencia, tecnología y sociedad en Latinoamérica: una interpretación política de su trayectoria”, Revista Iberoamericana de Ciencia, Tecnología y Sociedad, Centro REDES, Vol. III, N7:13-51.

Del Bello, Juan Carlos y Carlos Abeledo (2007) "Reflexiones sobre cuestiones pendientes de la Agenda de Política Pública en Ciencia, Tecnología e Innovación de Argentina", Ponencia presentada en Primer Congreso Argentino de Estudios Sociales de la Ciencia y la Tecnología, Bernal, Universidad Nacional de Quilmes.

Del Bello, Juan Carlos (2014) “Argentina: experiencias de transformación de la institucionalidad pública de apoyo a la innovación y al desarrollo tecnológico”, en Rivas, Gonzalo y Sebastián Rovira (eds.) Nuevas Instituciones para la innovación: Prácticas y experiencias en América Latina, Serie Documentos de Proyectos Comisión Económica para América Latina y el Caribe (CEPAL) N601:35-83.

Elzinga, Aant y Andrew Jamison (1996) "El cambio de las agendas políticas en ciencia y tecnología”, Revista Zona Abierta 75/76:91-133. 
Estevez, Alejandro M., Charles O. y James Anderson (2002) "El modelo secuencial de políticas públicas, treinta años más tarde”, Centro de Investigación en Administración Pública, Facultad de Ciencias Económicas, Universidad de Buenos Aires, Argentina.

Esteves, Alejandro O. y Susana Esper (2009) "Revisitando el modelo secuencial de políticas públicas. Sus etapas”. Revista del Instituto AFIP, 5:72-90.

Franco, Rolando y Jorge Lanzaro (coordinadores) (2006) Política y políticas públicas en los procesos de reforma de América Latina. Buenos Aires: Miño y Dávila.

Freeman, Christopher (1987) Technology policy and economic performance: lessons from Japan, London: Pinter Publishers.

García Palacios, Eduardo M.; Juan Carlos González, José Galbarte, J.A. López Cerezo, J.L. Lujan, N.M.Gordillo, C. Ossorio, C. y C. Valdez (2001) “Ciencia, Tecnología y Sociedad: una aproximación conceptual”, Cuadernos de Iberoamérica, OEI.

Gerston, Larry N. (2010) Public Policy Making: process and principles.USA: New York: M.E. Sharpe.

Gibbons, M. et al. (1997) La nueva producción del conocimiento: dinámica de la ciencia y la investigación en las sociedades contemporáneas. Barcelona: Pomares-Corredor.

Hall, Peter (1996) El Gobierno de la Economía, Ministerio de Trabajo y la Seguridad Social. Madrid: Alianza.

Herrera, Amicar (1971) Ciencia y política en América Latina. México: Siglo XXI.

Hurtado, Diego y Mallo, E. (2013) “Riesgos teóricos y agendas políticas de ciencia y tecnología: el "mal del modelo lineal" y las instituciones como cajas negras", en Andrés Kozel, Horacio Crespo y Héctor Palma (comps.). Heterodoxia y Fronteras en América Latina. Buenos Aires, Teseo-Universidad Autónoma del Estado de Morelos, :449-476.

Johnson, B. y Lundvall, B-A. (1994) "Sistemas Nacionales de Innovación y aprendizaje institucional”, Comercio Exterior,Vol. 44, N8:697.

Ladenheim, Ruth (2012) “Construcción Participativa de Políticas públicas en Ciencia, Tecnología e Innovación”, En Información de la Secretaría de Planeamiento y Políticas (INFOSePP), MINCyT, Año2, N³. http://en.mincyt.gob.ar/adjuntos/ archivos/000/024/0000024768.pdf, accesado el 1 de agosto 2014. 
Lahera, Eugenio (2004) Introducción a las políticas pública. Chile: Fondo de Cultura Económica.

López Cerezo, José Antonio (1998) "Ciencia,Tecnología y Sociedad: el estado de la cuestión en Europa y Estados Unidos”, en Revista Iberoamericana de Educación, OEI, Nº 18: 41-68.

\section{López Cerezo, José Antonio, M. González García y José Luis Lujan Lopez}

(1996) Ciencia, tecnología y sociedad. Una introducción al estudio social de la ciencia y la tecnología. Madrid:Tecno.

Lugones, Gustavo y Fernando Porta (2011) Investigación científica e innovación tecnológica en Argentina. Impacto de los fondos de la Agencia Nacional de Promoción Científica y Tecnológica. Bernal: Universidad Nacional de Quilmes.

Lundvall, B-A. (1992) National systems of innovation: towards a theory of innovation and interactive learning. London: Pinter.

Lundvall, B-A. (2002) National systems of production, innovation and competence building. Aalborg: Aalborg University, Department of Business Studies.

Mallo, Eduardo (2011) "Políticas de ciencia y tecnología en la Argentina: La diversificación de problemas globales, ¿¿soluciones locales?” REDES, vol. 17, N 32:133-160.

Miró, Julieta (s/f) "El ciclo de las políticas públicas”, Facultad de Trabajo Social, UNER, http://www.fts.uner.edu.ar/polit_planif/documentos/miro.htm, accesado el 1 de abril del 2015.

Nuñez Jover, J. (1999) “La ciencia y la tecnología como procesos sociales.

Lo que la educación científica no debería olvidar”, OEI: http://www.oei.es/salactsi/ nunez07.htm, accesado el 1 de abril del 2015.

OCDE (2011) “Hacia un mecanismo para el diálogo de políticas de innovación: Oportunidades y desafíos para América Latina y el Caribe”, París: Centro de Desarrollo de la OCDE.

Oszlak, Oscar y Guillermo O`Donnell (2007) “Estado y políticas estatales en América Latina: Hacia una estrategia de investigación”, en Carlos Acuña (comp.) Lecturas sobre el Estado y las políticas públicas: Retomando el debate de ayer para fortalecer el actual, Buenos Aires: Proyecto de Modernización del Estado, 2007:555-584. http:// politicayplanificacionsocial.sociales.uba.ar/files/2012/04/Oszlak-O-y-ODonnellG-1984-Estado-y-Polticas-estatales-en-Amrica-Latina-Hacia-una-estrategia-deinvestigacin.pdf, accesado el 29 de agosto de 2017. 
Pallares, Francesc (1988) "Políticas públicas. Sistema político en acción”, Revista de Estudios Políticos, N62:141-168. http://cienciassociales.edu.uy/wp-content/uploads/ sites/4/2013/archivos/Pallares,\%20Francesc\%20(1988).pdf, accesado el 1 de abril de 2015.

Patel, Parimal y Keith Pavitt (1994) "National innovation systems: why are they are important, and how they might be measured and compared". Economics of Innovation and New Technology, N³:77-95.

Pressman, J.L. y A. Wildavsky (1973), en AguilarVillanueva; L., El estudio de las políticas públicas, Estudio Introductorio, México: Miguel Ángel Porrúa.

Rein, M., y F. Rabinowitz, F. (1978) "La implementación: una perspectiva teórica. Entre la intención y la acción”, en AguilarVillanueva, L., La implementación de las políticas públicas. México: Porrúa:147-184.

Revuelta Vaquero, Benjamín (2007) "La implementación de políticas públicas”, Díkaion, N¹6:135-156.

Rivas, Gonzalo. y Sebastián Rovira (eds.) (2014) Nuevas instituciones para la innovación Prácticas y experiencias en América Latina. Santiago de Chile: CEPAL. Serie Documentos de Proyectos N601:260.

http://repositorio.cepal.org/handle/11362/36797, accesado el 1 de abril de 2015.

Roca, Alejandra y Mariana Versino (2009) "Las políticas de ciencia y tecnología en la Argentina reciente (1983-2008): los discursos de gestión y las prácticas de evaluación", en Dossiê Sistemas Territoriais de Inovação e Políticas de CET na América Latina, Rev. de Adm. Fead-Minas, Vol. 6, №1/2:33-55.

Sábato, Juan y Natalio Botana (1975) “La ciencia y la Tecnología en el desarrollo futuro de América Latina”, en Sábato J. (Coord.) El pensamiento latinoamericano en la problemática ciencia-tecnología-desarrollo-dependencia. Buenos Aires: Paidós.

Sanz Merino, Noemí (2008) "La apropiación política de la ciencia: origen y evolución de una nueva tecnocracia”. Revista Iberoamericana de Ciencia, Tecnología y Sociedad, Vol.4 No 10:85123. http://www.scielo.org.ar/pdf/cts/v4n10/v4n10a07.pdf, accesado el 20 de julio de 2015 .

Tamayo Sáez, Manuel. (1997) “El análisis de las políticas públicas”, en Bañon, Rafael y Castillo, Ernesto (comps.) La nueva Administración Pública, Madrid:Alianza Universidad:281-312. 
Vaccarezza, Leonardo (1998) “Ciencia, Tecnología y Sociedad: el estado de la cuestión en América Latina”. Revista Iberoamericana de Educación OEI, N¹8:13-40.

Varsavsky, Oscar (1969) Ciencia, política y cientificismo, Buenos Aires: CEAL.

Varsavsky, Oscar (1971) Ciencia y Estilos de Desarrollo. Ciencia Nueva, Revista de Ciencia y Tecnología, No 13:38-39. http://blog.ciencianueva.com/, accesado el 1 de abril de 2015.

Zurbriggen, Cristina. y Mariana Gonzalez Lago (2010) Políticas de Ciencia, Tecnología e Innovación en los países del MERCOSUR. Serie Cuadernos del CEFIR, $\mathrm{N}^{\circ} 007$, Montevideo: CEFIR.

http://www20.iadb.org/intal/catalogo/PE/2011/07648.pdf

\section{Fuentes}

InfoLEG Promoción y Fomento de la Innovación Tecnológica, Ley № 23.877, Ministerio de Economía de la República Argentina, Centro de Documentación e Información. http://www.infoleg.gob.ar/infolegInternet/anexos/0-4999/277/norma.html, accesado el 1 de agosto 2014.

MINCYT (2006) Plan Estratégico Nacional de Ciencia, tecnología e Innovación "Bicentenario" (2006-2010), Ministerio de Educación, Ciencia y Tecnología de la Nación. http://www.mincyt.gob.ar/_post/descargar.php?idAdjuntoArchivo=22513, accesado el 1 de agosto 2014.

OCDE (2011) "Hacia un mecanismo para el diálogo de políticas de innovación: Oportunidades y desafios para América Latina y el Caribe”. Centro de Desarrollo de la OCDE., http://www.oecd.org/centrodemexico/47435448.pdf,Accesado el 16 de febrero 2015.

\section{Cómo citar este artículo:}

Castro Demiryi, Victoria (2019) "Políticas de promoción y estímulo de la innovación: La importancia de la implementación de instrumentos". Revista Perspectivas de Políticas Públicas Vol. 8 N`16: 263-297 